\title{
A randomised crossover trial of chest physiotherapy in non-cystic fibrosis bronchiectasis
}

\author{
M.P. Murray*, J.L. Pentland ${ }^{\#}$ and A.T. Hill*
}

ABSTRACT: Regular chest physiotherapy is advocated in non-cystic fibrosis bronchiectasis despite little evidence supporting its routine use. This study aimed to establish the efficacy of regular chest physiotherapy in non-cystic fibrosis bronchiectasis compared with no regular chest physiotherapy.

20 patients not practising regular chest physiotherapy were enrolled in a randomised crossover trial of 3 months of twice daily chest physiotherapy using an oscillatory positive expiratory pressure device compared with 3 months of no chest physiotherapy. The primary end-point was the Leicester Cough Questionnaire (LCQ). Additional outcomes included 24-h sputum volume, forced expiratory volume in $1 \mathrm{~s}$ (FEV1), forced vital capacity (FVC), forced expiratory flow at $25-75 \%$ of FVC (FEF25-75\%), maximum inspiratory pressure (MIP), maximum expiratory pressure (MEP), exercise capacity, sputum microbiology and St George's Respiratory Questionnaire (SGRQ). The treatment effect was estimated using the differences of the pairs of observations from each patient.

There was a significant improvement in all domains and total $L C Q$ score with regular chest physiotherapy (median (interquartile range) total score improvement 1.3 (-0.17-3.25) units; $\mathrm{p}=0.002)$. 24-h sputum volume increased significantly with regular chest physiotherapy (2) (06) $\mathrm{mL} ; \mathrm{p}=0.02)$, as did exercise capacity $(40(15-80) \mathrm{m} ; \mathrm{p}=0.001)$ and SGRQ total score $(7.77$ $(-0.99-14.5)$ unit improvement; $p=0.004)$. No significant differences were seen in sputum bacteriology, FEV1, FVC, FEF25-75\%, MIP or MEP.

Regular chest physiotherapy in non-cystic fibrosis bronchiectasis has small, but significant benefits.

\section{KEYWORDS: Bronchiectasis, physiotherapy}

$\mathrm{n}$ bronchiectasis there is abnormal permanent dilatation of the airways, and the normal mucociliary clearance mechanism is impaired $[1,2]$. There are excessive bronchopulmonary secretions and patients have a persistent cough, frequent infective exacerbations and a poor health-related quality of life (HRQoL) [3, 4].

Chest physiotherapy aims to mobilise secretions and facilitate effective expectoration, providing control of cough and improving airway clearance. It is widely advocated as a mainstay of management for this chronic disease [5]. However, to date, there are no randomised controlled trials of chest physiotherapy exclusively in patients with noncystic fibrosis bronchiectasis [6].

Several different techniques or regimens exist for airway clearance, such as postural drainage, autogenic drainage, the active cycle of breathing technique, positive expiratory pressure (PEP), oscillatory PEP devices and high-frequency chest wall percussion. Previous small studies in noncystic fibrosis bronchiectasis have compared various techniques and found no single method to be superior; however, patient preference for technique has varied $[7,8]$.

The aim of this randomised crossover study was to establish the efficacy of routine chest physiotherapy in non-cystic fibrosis bronchiectasis, comparing the effect of twice daily physiotherapy using an oscillatory PEP device with no chest physiotherapy in patients not previously practising regular chest physiotherapy.

\section{METHODS}

This was a randomised crossover trial of 3 months of twice daily chest physiotherapy

\section{AFFILIATIONS}

Depts of *Respiratory Medicine, and \#Physiotherapy (Respiratory Medicine), Royal Infirmary of Edinburgh, Edinburgh, UK.

\section{CORRESPONDENCE}

M.P. Murray

Dept of Respiratory Medicine

Royal Infirmary of Edinburgh

51 Little France Crescent

Edinburgh

EH16 4SA

UK

E-mail: maevemurray@hotmail.com

Received:

April 032009

Accepted after revision:

June 032009

First published online:

June 182009 
followed by a 1-month washout period compared with 3 months of no chest physiotherapy in adults with non-cystic fibrosis bronchiectasis not routinely practising chest physiotherapy from October 2007 to December 2008 (Clinical Trial Registration NCT00816309). Randomisation was determined by computer generation and the study was approved by the Lothian Research Ethics Committee (Edinburgh, UK). The primary outcome was patient perceived cough severity measured using the Leicester Cough Questionnaire (LCQ) [9]. Secondary outcomes included 24-h sputum volume, forced expiratory volume in $1 \mathrm{~s}$ (FEV1), forced vital capacity (FVC), forced expiratory flow at $25-75 \%$ of FVC (FEF25-75\%), maximum inspiratory pressure (MIP), maximum expiratory pressure (MEP), exercise capacity, sputum microbiology and St George's Respiratory Questionnaire (SGRQ) [10].

\section{Patients}

Patients were recruited from the bronchiectasis clinic, Royal Infirmary of Edinburgh. Inclusion criteria were: 1) a radiological diagnosis of bronchiectasis using a high-resolution computed tomography (HRCT) chest scan according to the features described by NAIDICH et al. [11]; 2) chronic sputum expectoration; 3) not carrying out regular chest physiotherapy (for the purposes of this study this was defined as less than two occasions per week); and 4) clinically stable disease (defined as no requirement for antibiotics in the 4 weeks preceding study entry). Exclusion criteria were: current smokers; ex-smokers of $\leqslant 2$ yrs or ex-smokers with a history of $\geqslant 10$ pack-yrs of smoking and emphysema on HRCT; primary diagnosis of asthma; cystic fibrosis (as defined by the presence of transmembrane conductance regulator sequence variants present on genotyping); and active sarcoidosis or active tuberculosis.

\section{Physiotherapy}

Chest physiotherapy was carried out using the oscillatory PEP device Acapella Choice ${ }^{\circledR}$ (Smiths Medical ASD Inc., Weston, MA, USA). Each patient was trained by the chest physiotherapist (J.L. Pentland) to complete three sets of the following cycle for each treatment session: 10 breaths (each inhaling to three quarters of the maximum inspiratory capacity then a 3-s breath hold followed by exhalation to functional residual capacity) followed by two to three forced expiratory techniques (huffs) or coughs. The frequency/resistance dial (range 1-5) was set at three for all participants. This setting was the maximum tolerated by all participants. Patients completed two treatment sessions each day (morning and evening, typical duration 20$30 \mathrm{~min}$ ). Compliance and occurrence of any adverse effects (specifically, any haemoptysis or increased use of short-acting bronchodilator therapy) were assessed using a diary card which was reviewed monthly during the treatment phase. Technique was reviewed by the chest physiotherapist at monthly intervals during the treatment phase and the devices were retained by the physiotherapist during the nontreatment phase.

\section{Other interventions}

\section{Exacerbations requiring antibiotic therapy}

An exacerbation was defined as a clinical deterioration with all of the following: increasing cough, increasing sputum volume and worsening sputum purulence [12]. All patients who experienced an exacerbation during the study were reviewed by the study doctor and received a 14-day course of antibiotics (prescribed according to sputum bacteriology culture and sensitivities). No additional chest physiotherapy was advised. All were reviewed following completion of antibiotics to ensure recovery.

\section{Routine therapy}

Any changes made to the patient's usual respiratory medication during the study period were noted.

\section{End-points}

The study design and assessment time-points are shown in figure 1 .

\section{Cough severity and HRQOL}

The LCQ and the SGRQ were completed. Both questionnaires have been validated to reflect impaired HRQoL in bronchiectasis $[13,14]$. The LCQ is a 19 item self-completed quality of life measure of the impact of cough severity [9]. It has three domains: physical (eight items), psychological (seven items) and social (four items). The total severity score ranges from 3 to 21 , with a lower score indicating a more severe cough. The minimal clinically important difference (MCID) for change is 1.3 units [15]. The SGRQ is a 50 item self-administered HRQoL questionnaire consisting of three components: symptoms (eight items), activity (16 items) and impacts (26 items). The total score ranges from $0-100$, with a higher score indicating a poorer HRQoL. The MCID for the SGRQ is 4 units [14]

\section{Sputum analysis}

The total volume of sputum expectorated over a 24-h period was collected in a sterile transparent gradated container. Qualitative and quantitative bacteriology was performed on a separate early morning sample [16].

\section{Pulmonary function tests}

The following pulmonary function tests were performed: FEV1, FVC, FEF25-75\%, MIP and MEP [17, 18]. The highest of three technically satisfactory measurements (within 10\%) was recorded for each.

\section{Incremental shuttle walk test}

An externally paced, 10-m incremental field walking test was performed [19].

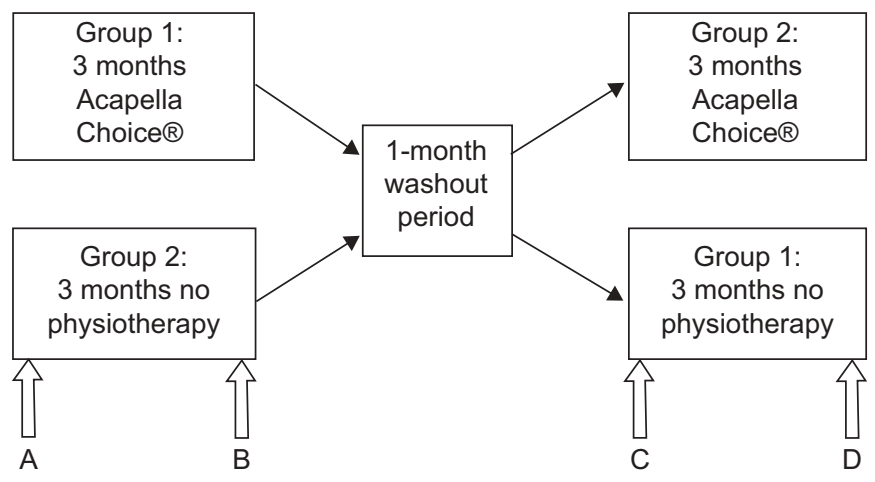

FIGURE 1. Study protocol. Assessments were performed at the start and end of each treatment period. A, B, C and D represent the assessment time-points. Acapella Choice ${ }^{2}$ : Smiths Medical ASD Inc., Weston, MA, USA. 


\section{Statistical analysis}

Statistical analysis was performed using SPSS for Windows, Version 17 (SPSS Inc., Chicago, IL, USA). The primary aim was to detect an improvement in LCQ score. Using the original validation of the LCQ, with 20 patients, a 5\% level of significance (two tailed), a common SD of 0.94 and a power of $80 \%$ would detect a mean difference in LCQ score of 0.63 [9]. Using the validation of the LCQ in non-cystic fibrosis bronchiectasis, with 20 patients, a 5\% level of significance (two tailed), a common SD of 1.1 and a power of $80 \%$ would detect a mean difference in LCQ score of 0.73 [13]. Data was analysed according to the method of JONES and KENWARD [20]. The treatment effect was estimated using the differences of the matched pairs of observations from each patient and are presented as median (interquartile range). Treatment differences between regular chest physiotherapy and no chest physiotherapy were compared using the Wilcoxon test. A two-tailed $\mathrm{p}$-value of $<0.05$ was considered significant.

\section{RESULTS \\ Patients}

In total, 20 outpatients were recruited. The baseline demographics and patient characteristics are detailed in table 1.

\section{Study entry and post-washout}

The baseline characteristics for the end-points at entry to each phase of the study are detailed in table 2. There was no significant difference in total LCQ score, 24-h sputum volume,

\section{TABLE 1 Patient characteristics}

\begin{tabular}{lc} 
Study participants ${ }^{\#}$ & 20 \\
Male & $12(60)$ \\
Age yrs & $73(72-77)$ \\
Ex-smokers & $8(40)$ \\
Chronic cardiac disease & $3(15)$ \\
Neurological disease & $1(5)$ \\
Chronic renal impairment & $1(5)$ \\
Diabetes mellitus & 0 \\
Inhaled corticosteroid therapy & $12(60)$ \\
Systemic corticosteroid therapy & 0 \\
Long-term antibiotic therapy & $2(10)$ \\
Infective exacerbations requiring antibiotic treatment in & $2(1.5-3)$ \\
$\quad$ preceding 12 months & \\
Lobes affected with bronchiectasis on HRCT & $4(3-4.75)$ \\
Varicose or cystic dilatation affecting $\geqslant \mathbf{1}$ lobe & $15(75)$ \\
Chronically colonised with pathogenic organisms in & $14(70)$ \\
$\quad$ sputum when stable & \\
Pseudomonas aeruginosa & $6(42.9)$ \\
Haemophilus influenzae & $5(35.7)$ \\
Staphylococcus aureus & $2(14.3)$ \\
Moraxella catarrhalis & $1(7.1)$ \\
Aetiology of bronchiectasis & \\
Post-infective & $10(50)$ \\
Idiopathic & $8(40)$ \\
Inactive allergic bronchopulmonary aspergillosis & $1(5)$ \\
Inflammatory bowel disease & $1(5)$ \\
\hline
\end{tabular}

Data are presented as $\mathrm{n}, \mathrm{n}$ (\%) or median (interquartile range). HRCT: high-

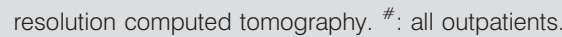

FEV1, FVC, FEF25-75\%, MIP, MEP, exercise capacity, sputum microbiology and SGRQ score (table 2) between entry to the first arm of the study (fig. 1, point A) and at the end of the washout period (fig. 1, point C), prior to entry to the second arm of the study.

\section{Completion and adverse events}

All patients completed the study and no adverse effects with the oscillatory PEP device occurred. There were 12 exacerbations affecting 11 patients during the study period (table 3). No other interventions or changes to patients' care were required throughout the duration of the study.

\section{Treatment differences}

There was a significant improvement in all domains of the LCQ and total score (table 3, fig. 2).

24-h sputum volume significantly increased with regular chest physiotherapy compared with no chest physiotherapy (fig. 3). The total SGRQ score improved significantly with regular chest physiotherapy but the only significant improvement seen in the individual domains of the SGRQ score was in the activity domain (fig. 4). Exercise capacity also improved significantly with regular chest physiotherapy (fig. 5) but there were no significant differences seen in sputum bacteriology, FEV1, FVC, FEF25-75\%, MIP or MEP, or exacerbation frequency (table 3).

\section{DISCUSSION}

This randomised crossover trial found that twice daily chest physiotherapy in patients with non-cystic fibrosis bronchiectasis not normally practising regular physiotherapy, significantly improved perceived cough severity, increased 24-h sputum volume, improved exercise capacity and SGRQ score, but had no effect on sputum microbiology, FEV1, FVC, FEF25$75 \%$, MIP, MEP or exacerbation frequency.

Clearance of bronchopulmonary secretions is impaired in patients with bronchiectasis [2]. MAZZAOCCO et al. [21] first explored the potential benefits of chest physiotherapy in bronchiectasis patients over 20 yrs ago, but despite further studies investigating various physiotherapy techniques to aid clearance, there have been no randomised controlled studies exploring the efficacy of regular chest physiotherapy. The primary outcome measured was selected to reflect one of the major goals of management of chronic disease: an improvement in HRQoL. Specifically, we wished to assess the impact of the predominant symptom of bronchiectasis, cough severity.

Our study population had clinically significant bronchiectasis. They had an average of $2(1.5-3)$ exacerbations in the preceding 12 months, over two-thirds were chronically colonised with pathogenic organisms in their sputum and, radiologically, 4 (34.75) lobes were affected with bronchiectasis on computed tomography chest scan and $75 \%$ had varicose or cystic dilatation in at least one lobe. Despite this, they did not carry out regular chest physiotherapy prior to the study.

Our study was conducted with twice daily chest physiotherapy over 3 months as an outpatient. We selected the Acapella Choice ${ }^{\circledR}$ device as the airway clearance technique both for ease of use and based on patient preference from previous studies $[8,22]$. Currently, there is no clear evidence for the optimum frequency or duration of airway clearance. 
TABLE 2 Patient characteristics at entry to each study phase

\begin{tabular}{|c|c|c|c|}
\hline Characteristic & Start of study ${ }^{\#}$ & End of washout period & p-value \\
\hline Total LCQ score ${ }^{+}$ & $16.3(14.1-17.9)$ & $15.9(13.8-19.4)$ & 0.5 \\
\hline FEV1 L & $1.68(1.25-2.31)$ & $1.72(1.19-2.10)$ & 0.8 \\
\hline FEV $1 \%$ pred & $75.7(48.3-98.1)$ & $68.4(53-107.1)$ & \\
\hline FVC L & $2.64(1.9-3.65)$ & $2.82(1.75-3.5)$ & 0.6 \\
\hline FEV $1 /$ FVC $\%$ pred & $87.1(77.6-104.4)$ & $97.0(76.1-120)$ & \\
\hline FEF $25-75 \% L \cdot s^{-1}$ & $0.95(0.64-1.54)$ & $1.09(0.54-1.84)$ & 0.96 \\
\hline FEF25-75\% \% pred & $47.5(21.9-64.8)$ & $44.8(24.8-96.4)$ & \\
\hline MIP $\mathrm{cmH}_{2} \mathrm{O}$ & $43.5(33.2-72.5)$ & $48(32.5-61.5)$ & 0.2 \\
\hline MIP \% pred & $58.5(37.2-77.2)$ & 51.7 (31.1-63) & \\
\hline MEP $\mathrm{cmH}_{2} \mathrm{O}$ & $68.5(58.5-95.2)$ & $67(51-109)$ & 0.3 \\
\hline Total SGRQ score s $^{\S}$ & $41.1(24.6-44.8)$ & $40.4(18.0-52.5)$ & 0.6 \\
\hline
\end{tabular}

However, it is recognised that airway clearance regimens need to be effective without affecting other activities of daily living. We selected a twice daily physiotherapy regimen to account for this and also because previous studies assessing different physiotherapy techniques for non-cystic fibrosis bronchiectasis have achieved compliance with this frequency $[8,23]$. Further studies are needed to specifically address the optimal frequency and duration of physiotherapy.

Several adjuncts to physiotherapy currently exist including: 1) bronchodilator therapy which may minimise bronchial hyperreactivity and improve airway clearance; 2) inhaled hyperosmolar agents (nebulised hypertonic saline has been shown to yield greater sputum weights and inhaled mannitol has been shown in small studies to aid mucociliary clearance [24, 25]); and 3) pulmonary rehabilitation and inspiratory muscle training has previously been shown to improve exercise tolerance [26]. However, inhaled mucolytics (recombinant human DNase), although of benefit in cystic fibrosis, are not recommended in non-cystic fibrosis bronchiectasis because they have a significant negative impact on FEV1 [27]. Our study did not employ any such adjuncts. Further studies are

\section{TABLE 3 Treatment differences}

\begin{tabular}{|c|c|c|c|}
\hline Total LCQ score improvement & $1.3(-0.17-3.25)$ & $0(-1.5-0.5)$ & 0.002 \\
\hline FEV1 L & $-0.01(-0.06-0.08)$ & $-0.01(-0.1-0.11)$ & 0.7 \\
\hline FVC L & $-0.01(-0.09-0.28)$ & $0.06(-0.08-0.21)$ & 0.9 \\
\hline FEF $25-75 \% L \cdot s^{-1}$ & $-0.02(-0.17-0.16)$ & $0.04(-0.1-0.34)$ & 0.6 \\
\hline Exercise capacity $m$ & $40(15-80)$ & $0(-10-20)$ & 0.001 \\
\hline Sputum bacterial load cfu $\cdot \mathrm{mL}^{-1}$ & $-1 \times 10^{3}\left(-2.78 \times 10^{6}-1.74 \times 10^{7}\right)$ & $1 \times 10^{3}\left(-6.5 \times 10^{7}-6.4 \times 10^{6}\right)$ & 0.72 \\
\hline Total SGRQ score improvement & $7.8(-0.99-14.5)$ & $-0.7(-2.3-0.05)$ & 0.005 \\
\hline Exacerbations $\mathbf{n}$ & 5 & 7 & 0.48 \\
\hline
\end{tabular}




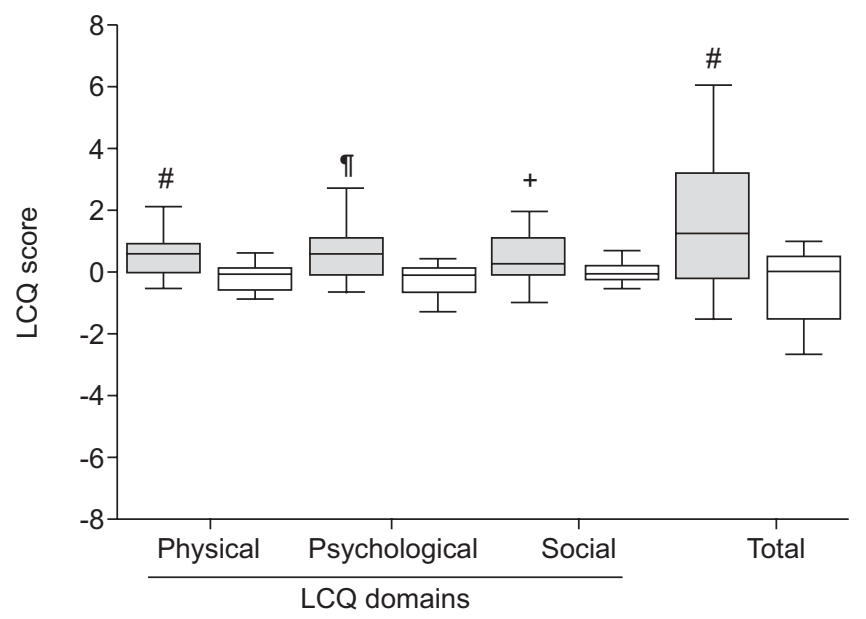

FIGURE 2. Change in Leicester Cough Questionnaire (LCQ) individual domain and total scores with physiotherapy $(\square)$ and with no physiotherapy $(\square)$. The horizontal lines represent the median and interquartile ranges for each group and the whiskers represent the maximum and minimum. ${ }^{*}: p=0.002 ;{ }^{\top}: p<0.0001$; $+\mathrm{r}=0.02$

needed to assess efficacy of such techniques in addition to regular chest physiotherapy.

This study did not have a sham arm and, instead, we compared twice daily chest physiotherapy using an oscillatory PEP device with no chest physiotherapy. This design was intentional as any type of sham would involve some form of airway clearance. A limitation of this study design, however, is the potential for a placebo effect. The crossover design was used to offer all patients a period of regular chest physiotherapy during the study. There was no carry over effect with the 1-month washout phase.

One of the main goals of long-term management of chronic respiratory disease is to improve HRQoL and with regular,

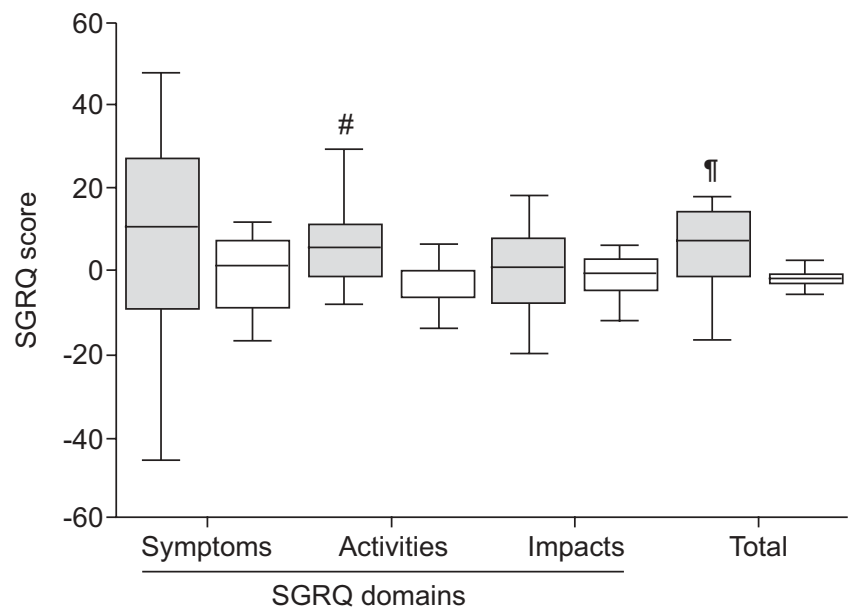

FIGURE 4. Change in St George's Respiratory Questionnaire (SGRQ) individual domain and total scores with physiotherapy $(\square)$ and with no physiotherapy $(\square)$. The horizontal lines represent the median and interquartile ranges for each group and the whiskers represent the maximum and minimum. \#: $p=0.02 ;{ }^{\circ}: p=0.005$

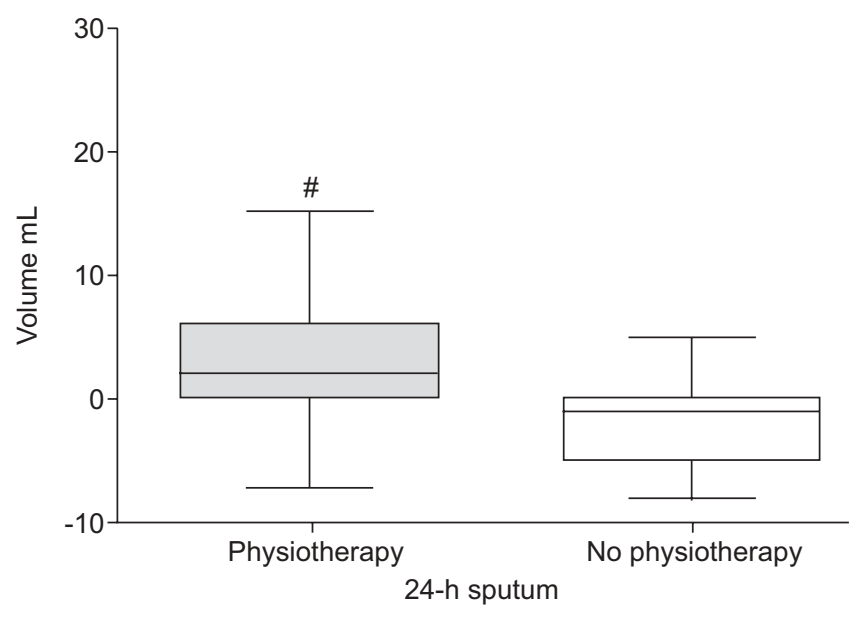

FIGURE 3. Change in 24-h sputum volume with physiotherapy and with no physiotherapy. The horizontal lines represent the median and interquartile ranges and the whiskers represent the maximum and minimum. ${ }^{*}: p=0.02$.

controlled airway clearance, patient perception of cough severity improved with an increase in score seen in all domains of the LCQ. Since the completion of this study, a 1.3 unit difference in the total LCQ score has been established as a clinically significant change and we observed a median increase of 1.3 units following 3 months of twice daily chest physiotherapy [15]. An open label study by MUTALITHAS et al. [28] found a mean improvement of 3.1 units in total LCQ score with bronchopulmonary hygiene physical therapy. Importantly, although our study was not powered to detect a change in SGRQ score, we observed that this too improved, with the total score improving beyond its established minimal clinically significant change of 4 units (median improvement of 7.8 units) [14].

A major rationale for chest physiotherapy is to loosen secretions and enhance expectoration. According to previous work by CECINS et al. [29], our study was sufficiently powered to detect a $15 \%$ change in 24 -h sputum volume and we found that with

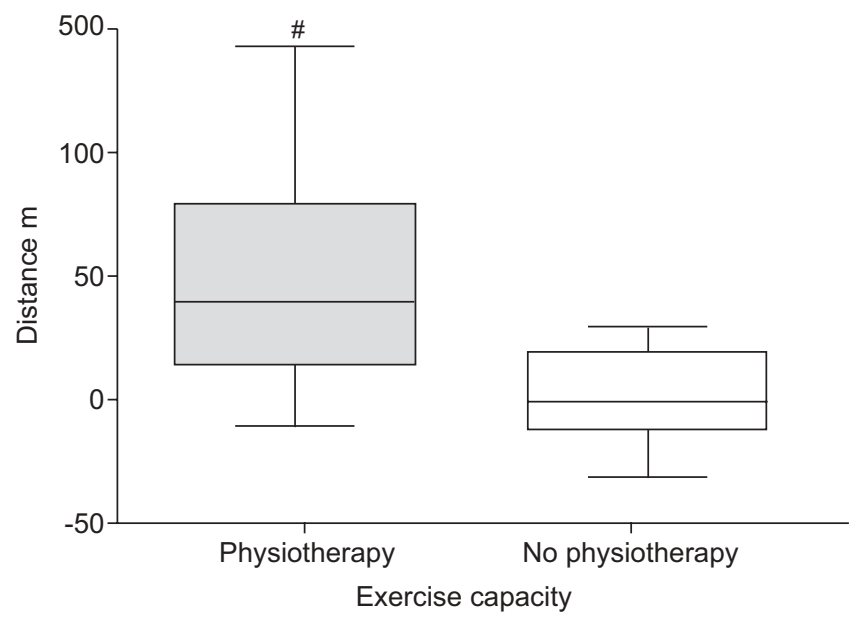

FIGURE 5. Change in exercise capacity with physiotherapy and with no physiotherapy. The horizontal lines represent the median and interquartile ranges for each group and the whiskers represent the maximum and minimum \#: $p=0.001$. 
twice daily chest physiotherapy the mean volume of sputum expectorated over $24 \mathrm{~h}$ increased by $2(0-6) \mathrm{mL}$. Although the value of 24-h sputum collections in clinically stable outpatients may be limited by patient compliance and confounded by factors such as swallowed secretions, it is a highly pertinent, noninvasive marker and has been selected as a relevant outcome measure in previous studies assessing other potential long-term therapeutic strategies in bronchiectasis, including inhaled steroids and long-term antibiotics [30-33].

We also observed an increase in the distance achieved during the incremental shuttle walk test following 3 months of twice daily chest physiotherapy. Based on a previous study by NeWALl et al. [26], our patient sample size was powerful enough to detect a significant effect on exercise capacity. This perhaps emphasises the improvement in the activities domain of the SGRQ and that with greater control of cough and clearance of mucus from the airways, exercise capacity also improves.

The benefits observed with regular chest physiotherapy were small however, and we did not find any improvements in the remaining study end-points. Despite increased sputum expectoration, we observed no change in sputum bacterial load or any improvement in FEV1, FVC, FEF25-75\%, MIP, MEP or exacerbation frequency. One previous study of airway clearance techniques in bronchiectasis comparing the Flutter device with the active cycle of breathing technique found a significant improvement in FEV1 (0.08 L, 95\% CI 0.01-0.15 using the Flutter device); however, this improvement in FEV1 was not thought to be clinically significant [8]. Our patient cohort had a mean age of 73 (72-77) yrs and with increasing age there is less airway reversibility, perhaps further limiting the opportunity for any benefit from chest physiotherapy on FEV1 or FVC. In addition, we found no improvements in the assessment of small airways or inspiratory and expiratory pressures. However, this may be due to the intrinsic variability of such measurements. A previous study by NeWALL et al. [26] in noncystic fibrosis bronchiectasis patients found MEP to be impaired but MIP were within normal range. Our study population was, however, older which may explain the difference. Despite the lack of improvements in these measures, exercise capacity significantly increased.

In conclusion, this randomised crossover study found that regular chest physiotherapy in non-cystic fibrosis bronchiectasis has significant benefits compared with no chest physiotherapy. Despite the differences being small, achieving an improvement in functional ability and HRQoL is highly relevant to the management of this long-term illness. Larger studies are needed to explore potential benefits on other outcome measures.

\section{CLINICAL TRIALS}

This study is a registered on the Clinical Trials Database at Clinicaltrials.gov (NCT00816309). It is also registered on the National Research Registry (N0519192394)

\section{SUPPORT STATEMENT}

This study was funded by a Small Project Grant (NHS Lothian Research and Development Fund, Edinburgh, UK). M. Murray is funded by the Chief Scientist Office (Edinburgh).

\section{STATEMENT OF INTEREST}

None declared.

\section{ACKNOWLEDGEMENTS}

We would like to thank I. Pavord (Institute for Lung Health, University of Leicester, Leicester, UK) and S. Birring (King's college Hospital, London, UK) for their kind permission to use the Leicester Cough Questionnaire and P. Jones (St George's Hospital, London, UK) for his kind permission to use the St George's Respiratory Questionnaire. We would also like to thank the staff of the Pulmonary Function Laboratory, Royal Infirmary of Edinburgh, Edinburgh, UK and D. McAllister (Royal Infirmary of Edinburgh) for his assistance with the design of the study.

\section{REFERENCES}

1 Laennec RTH, Forbes J, eds. A Treatise in the Diseases of the Chest and on Mediate Auscultation. 4th Edn. Longman, London, 1834.

2 Currie DC, Pavia D, Agnew JE, et al. Impaired tracheobronchial clearance in bronchiectasis. Thorax 1987; 42: 126-130.

3 King PT, Holdsworth SR, Freezer NJ, et al. Characterisation of the onset and presenting clinical features of adult bronchiectasis Respir Med 2006; 100: 2183-2189.

4 Martinez-Garcia MA, Perpina-Tordera M, Roman-Sanchez P, et al. Quality-of-life determinants in patients with clinically stable bronchiectasis. Chest 2005; 128: 739-745.

5 O'Neill B, Bradley JM, McArdle N, et al. The current physiotherapy management of patients with bronchiectasis: a UK survey. Int J Clin Pract 2002; 56: 34-35.

6 Jones AP, Rowe BH. Bronchopulmonary hygiene physical therapy for chronic obstructive pulmonary disease and bronchiectasis. Cochrane Database Syst Rev 2000: 2; CD000045.

7 Eaton T, Young P, Zeng I, et al. A randomized evaluation of the acute efficacy, acceptability and tolerability of flutter and active cycle of breathing with and without postural drainage in noncystic fibrosis bronchiectasis. Chron Respir Dis 2007; 4: 23-30.

8 Thompson CS, Harrison S, Ashley J, et al. Randomised crossover study of the Flutter device and the active cycle of breathing technique in non-cystic fibrosis bronchiectasis. Thorax 2002; 57 446-448.

9 Birring SS, Prudon B, Carr AJ, et al. Development of a symptom specific health status measure for patients with chronic cough Leicester Cough Questionnaire (LCQ). Thorax 2003; 58: 339-343.

10 Jones PW, Quirk FH, Baveystock CM, et al. A self-complete measure of health status for chronic airflow limitation. The St. George's Respiratory Questionnaire. Am Rev Respir Dis 1992; 145: 1321-1327.

11 Naidich DP, McCauley DI, Khouri NF, et al. Computed tomography of bronchiectasis. J Comput Assist Tomogr 1982; 6: 437-444.

12 Anthonisen NR, Manfreda J, Warren CP, et al. Antibiotic therapy in exacerbations of chronic obstructive pulmonary disease. Ann Intern Med 1987; 106: 196-204.

13 Murray MP, Turnbull K, Macquarrie S, et al. Validation of the Leicester Cough Questionnaire in non-cystic fibrosis bronchiectasis. Eur Respir J 2009: 34; 125-131.

14 Wilson CB, Jones PW, O'Leary CJ, et al. Validation of the St George's Respiratory Questionnaire in bronchiectasis. Am J Respir Crit Care Med 1997; 156: 536-541.

15 Raj AA, Pavord DI, Birring SS. Clinical cough IV: what is the minimal important difference for the Leicester Cough Questionnaire? Handb Exp Pharmacol 2009; 187: 311-320.

16 Pye A, Stockley RA, Hill SL. Simple method for quantifying viable bacterial numbers in sputum. J Clin Pathol 1995; 48: 719-724.

17 Pellegrino R, Viegi G, Brusasco V, et al. Interpretative strategies for lung function tests. Eur Respir J 2005; 26: 948-968. 
18 Black LF, Hyatt RE. Maximal respiratory pressures: normal values and relationship to age and sex. Am Rev Respir Dis 1969; 99: 696-702.

19 Revill SM, Morgan MD, Singh SJ, et al. The endurance shuttle walk: a new field test for the assessment of endurance capacity in chronic obstructive pulmonary disease. Thorax 1999; 54: 213-222.

20 Jones B, Kenward MG, eds. Design and Analysis of Cross-over Trials. Chapman and Hall, New York, 1989.

21 Mazzocco MC, Owens GR, Kirilloff LH, et al. Chest percussion and postural drainage in patients with bronchiectasis. Chest 1985; 88: 360-363.

22 Currie DC, Munro C, Gaskell D, et al. Practice, problems and compliance with postural drainage: a survey of chronic sputum producers. Br J Dis Chest 1986; 80: 249-253.

23 Patterson JE, Hewitt O, Kent L, et al. Acapella versus "usual airway clearance" during acute exacerbation in bronchiectasis: a randomized crossover trial. Chron Respir Dis 2007; 4: 67-74.

24 Kellett F, Redfern J, Niven RM. Evaluation of nebulised hypertonic saline $(7 \%)$ as an adjunct to physiotherapy in patients with stable bronchiectasis. Respir Med 2005; 99: 27-31.

25 Daviskas E, Anderson SD, Eberl S, et al. Effect of increasing doses of mannitol on mucus clearance in patients with bronchiectasis. Eur Respir J 2008; 31: 765-772.
26 Newall C, Stockley RA, Hill SL. Exercise training and inspiratory muscle training in patients with bronchiectasis. Thorax 2005; 60: 943-948.

27 O'Donnell AE, Barker AF, Ilowite JS, et al. Treatment of idiopathic bronchiectasis with aerosolized recombinant human DNase I rhDNase Study Group. Chest 1998; 113: 1329-1334.

28 Mutalithas K, Watkin G, Willig B, et al. Improvement in health status following bronchopulmonary hygiene physical therapy in patients with bronchiectasis. Respir Med 2008; 102: 1140-1144.

29 Cecins NM, Jenkins SC, Pengelley J, et al. The active cycle of breathing techniques - to tip or not to tip? Respir Med 1999; 93: 660-665.

30 Tsang KW, Tan KC, Ho PL, et al. Inhaled fluticasone in bronchiectasis: a 12 month study. Thorax 2005; 60: 239-243.

31 Tsang KW, Ho PI, Chan KN, et al. A pilot study of low-dose erythromycin in bronchiectasis. Eur Respir J 1999; 13: 361-364.

32 el-Din MA, Palmer LB, el-Tayeb MN, et al. Nebulizer therapy with antibiotics in chronic suppurative lung disease. J Aerosol Med 1994; 7: $345-350$.

33 Cymbala AA, Edmonds LC, Bauer MA, et al. The diseasemodifying effects of twice-weekly oral azithromycin in patients with bronchiectasis. Treat Respir Med 2005; 4: 117-122. 